International Journal of Applied Mathematical Research, 1 (2) (2012) 117-129

(C) Science Publishing Corporation

www.sciencepubco.com/index.php/IJAMR

\title{
Predator-prey Model with Different Growth Rates and Different Functional Responses: A Comparative Study with Additional Food
}

\author{
Banshidhar Sahoo \\ Department of Mathematics, Daharpur A.P.K.B Vidyabhaban \\ Paschim Medinipur, West Bengal, India. \\ Email:banshivu@gmail.com
}

\begin{abstract}
A predator-prey model with different types of growth rates and different functional responses is proposed and analysed. The stable dynamics of the system for different growth rates have been shown. The results of the system with additional food to predator for different growth rates have been shown in this context. The coexistence of species in the system depends on the growth rates of the prey species. We have suggested that a real predator-prey model will be constructed with different growth rates and different functional responses.
\end{abstract}

Keywords: Predator-prey, Growth rates, Functional responses, Global stability, Additional food.

\section{Introduction}

The well known Verhulst logistic equation [1] for population dynamics is

$$
\frac{d N}{d t}=r N\left(1-\frac{N}{K}\right)
$$

where $r$ is the intrinsic growth rate of the population and $K$ is the carrying capacity. This model is parameterized by the initial population size, the initial growth rate and carrying capacity $K$. The resulting logistic growth rate curve is sigmoidal, when the population size is less than $K$. Even, the point of inflection for the system is fixed for which corresponding population is $\frac{K}{2}$. 


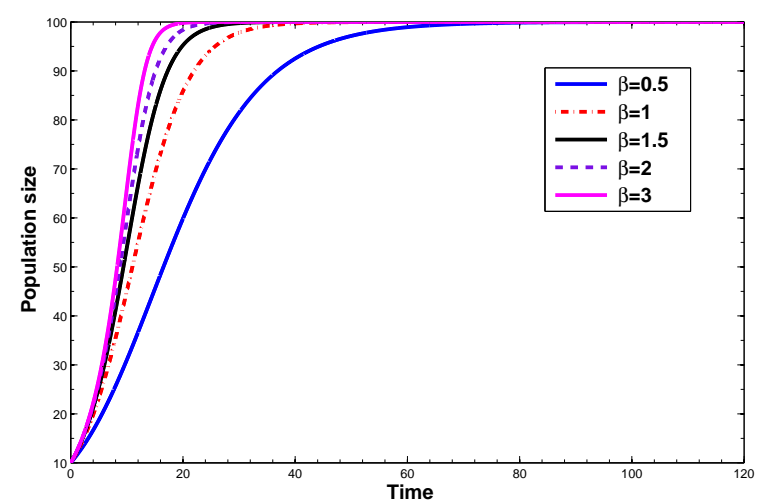

Figure 1: Population growth curve in time according to Richards' equation (1) for $r=0.2, K=100$.

This limitation is undesirable as well as unrealistic. In spite of this limitation, we see that there are several models where this logistic equation is used. The logistic growth equation has been used in many diverse biological systems $[2$, 3]. Morgan [4] used this logistic equation to describe herding behaviour of African elephant. Out side of the biology, Fisher and Pry [5] have successfully applied the logistic growth model to describe the market penetration of many new products and technologies. An evolutionary process in the industrial revolution may also be modelled with logistic equation is shown by Herman and Montroll [6]. However, the verhulst logistic equation is also referred to the literature as the Verhulst-Pearl equation after Verhulst [1], who first derived the curve, and Pearl [7], who used the curve to approximate population growth in the United States in 1920.

Since the original work of Verhulst [1], Pearl and Reed [7] there have been proposed several contributions suggesting alternative growth functional forms, whilst retaining the sigmoid and asymptotic property of the Verhulst logistic curve [1]. Richards [8] was the possibly first to apply a growth equation developed first by Von Bertalanffy [9] to describe the growth of animals. Richards [8] suggested the growth equation in the following form:

$$
\frac{d N}{d t}=r N\left[1-\left(\frac{N}{K}\right)^{\beta}\right]
$$

where $\beta$ is positive exponent and the term $\beta$ is consider as intraspecific com- 
petition factor. The solution of the equation (1) is

$$
N(t)=K\left[1-e^{-\beta r t}\left[1-\left(\frac{N_{0}}{K}\right)^{-\beta}\right]\right]^{-\frac{1}{\beta}}
$$

In particular, $\beta=1$ it reduces to the Verhulst growth equation [1]. The figure 1 illustrates the Richards' growth curve for different values of $\beta$ with $r=0.2, K=100$. Fig 1 depicts that the larger $\beta$ the faster in time the curve reaches the carrying capacity $K$. Richards growth curve was used for fitting experimental data by Nelder [10], who used the term generalized logistic equation to describe the equation.

In ecology there are huge number of food chain models with two, three or more species with different functional responses such as Holling-Turner type $[11,12]$, Beddington-DeAngelis type $[13,14]$ and ratio dependent type $[15,16]$ etc. But, most of the models have same type of growth rate and functional response. From biological point of view, it is unrealistic in nature. In fact, in the real world, predators feed on diffrent preys in different types of consumption ways. For example, consider crops, aphids, and birds as prey, another prey, and predator, respectively. In this case, it is natural to assume that the feeding type of birds on crops is different from that of birds on aphids. Therefore, to describe this phenomenon, two different types of functional responses are needed in the model. So, in this paper, we consider two types of functional responses, one the Holling type-II and other Beddington-DeAngelis functional response.

We shall now concentrate to formulate a food chain model with two preys and one predator species. In real world, the growth rate of different species is different. So we consider two prey species, one with Verhulst logistic growth equation [1] and other with Richards growth equation [8] with two types of functional responses. With above considerations, we formulate a food chain model in the form:

$$
\begin{aligned}
\frac{d X}{d T} & =R_{1} X\left(1-\frac{X}{K_{1}}\right)-\frac{A_{1} X Z}{B_{1}+X} \\
\frac{d Y}{d T} & =R_{2} Y\left[1-\left(\frac{Y}{K_{2}}\right)^{\beta}\right]-\frac{A_{2} Y Z}{B_{2}+Y+Z} \\
\frac{d Z}{d T} & =\frac{E_{1} A_{1} X Z}{B_{1}+X}+\frac{E_{2} A_{2} Y Z}{B_{2}+Y+Z}-D Z
\end{aligned}
$$

where $X$ and $Y$ are the density of prey species and $Z$ be the number of predator species that preys upon both $X$ and $Y$. T is the time. The constants $R_{1}, R_{2}$ and $K_{1}, K_{2}$ are the intrinsic growth rate and carrying capacity of $X$ and $Y$ 
respectively. $A_{1}, A_{2}$ are respectively the food intake rate of the predator $Z$ on $X$ and $Y$. The constants $E_{1}$ and $E_{2}$ are the conversion rates of preys $X$ and $Y$ to predator $Z$ respectively. $B_{1}$ and $B_{2}$ are the half saturation constants for the predator $Z$. The term $D$ denotes the predator's death rate.

We nondimensionalize the system (2) using $x=\frac{X}{K_{1}}, y=\frac{Y}{K_{2}}, z=\frac{Z}{K_{1} K_{2}}$, $t=R_{1} R_{2} T$ and we obtain the following system

$$
\begin{aligned}
& \frac{d x}{d t}=a x(1-x)-\frac{p x z}{1+b_{1} x} \\
& \frac{d y}{d t}=b y\left(1-y^{\beta}\right)-\frac{q y z}{1+b_{2} y+b_{3} z} \\
& \frac{d z}{d t}=\frac{\epsilon_{1} p x z}{1+b_{1} x}+\frac{\epsilon_{2} q y z}{1+b_{2} y+b_{3} z}-d z
\end{aligned}
$$

where $a=\frac{1}{R_{2}}, b=\frac{1}{R_{1}}, p=\frac{A_{1} K_{1} K_{2}}{R_{1} R_{2} B_{1}}, q=\frac{A_{2} K_{1} K_{2}}{R_{1} R_{2} B_{2}}, b_{1}=\frac{K_{1}}{B_{1}}, b_{2}=\frac{K_{2}}{B_{2}}, b_{3}=\frac{K_{1} K_{2}}{B_{2}}$, $\epsilon_{1}=\frac{E_{1}}{K_{2}}, \epsilon_{2}=\frac{E_{2}}{K_{1}}, d=\frac{D}{R_{1} R_{2}}$. The system (3) has to be analyzed with the following initial conditions: $x(0)>0, y(0)>0, z(0)>0$.

The main objective of this paper is to investigate the dynamics of the system (3). We have discussed the dissipativeness and condition for existence of global stability of the system (3) in section 2. The effects of additional food is discussed in section 3. The numerical simulation is shown in secion 4 . Finally, the conclusion is given in section 5 .

\section{Preliminary Notes}

\subsection{Dissipativeness}

Obviously the interaction functions of the system (3) are continuous and have continuous partial derivatives on $R_{+}^{3}=\left\{(x, y, z)^{T}: x(t)>0, y(t)>\right.$ $0, z(t)>0\}$. Therefore the solution of the model system (3) with positive initial condition exists and is unique, as the solution of system (3) initiating in the positive octant is bounded. Further more, the system (3) is said to be dissipative if all population initiating in the space $R_{+}^{3}=\left\{(x, y, z)^{T}: x(t)>\right.$ $0, y(t)>0, z(t)>0\}$ are uniformly limited by their environment [17].

Theorem-1: The system (3) is dissipative.

Proof: From the first equation of the system (3), we have

$\frac{d x}{d t} \leq a x(1-x) \Rightarrow x(t) \leq \frac{1}{1-C e^{-a t}}$, for all $t \geq 0$, with $C=1-\frac{1}{x_{0}}$, which implies that $x(t)<1$ for sufficiently large $t$. 
Let $(x(t), y(t), z(t))$ be any solution of the system (3) with positive initial conditions and define that $w=\epsilon_{1} x+\epsilon_{2} y+z$

i.e, $\quad \frac{d w}{d t}=\epsilon_{1} \frac{d x}{d t}+\epsilon_{2} \frac{d y}{d t}+\frac{d z}{d t}$

Therefore, $\frac{d w}{d t}=\epsilon_{1}\left[a x(1-x)-\frac{p x y}{1+b_{1} x}\right]+\epsilon_{2}\left[b y\left(1-y^{\beta}\right)-\frac{q y z}{1+b_{2} y+b_{3} z}\right]+\frac{\epsilon_{1} p x y}{1+b_{1} x}+\frac{\epsilon_{2} q y z}{1+b_{2} y+b_{3} z}-d z$.

Therefore,

$$
\begin{aligned}
\frac{d w}{d t} & =\epsilon_{1} a x(1-x)+\epsilon_{2} b y\left(1-y^{\beta}\right)-d z \\
i . e, \frac{d w}{d t} & \leq a \epsilon_{1}-\theta(x+y+z)
\end{aligned}
$$

where $\theta=\min \left\{a \epsilon_{1},-b \epsilon_{2}, d\right\}>0$.

$$
\frac{d w}{d t}+\theta w \leq a \epsilon_{1}
$$

Applying the theory of differential inequality we obtain $0<w<\frac{a \epsilon_{1}\left(1-e^{-\theta t}\right)}{\theta}+w(x(0), y(0), z(0)) e^{-\theta t}$

For $t \rightarrow \infty$, we have $0<w<\frac{a \epsilon_{1}}{\theta}$.

Hence all the solutions of the system (3) that initiate in $R_{+}^{3}$ are confined in the region

$S=\left\{(x, y, z) \in R_{+}^{3}: w=\frac{a \epsilon_{1}}{\theta}+\eta\right.$, for any $\left.\eta>0\right\}$, which means that all species are uniformly bounded for any initial value in $R_{+}^{3}$.

According to the above theorem we assume that there exists real numbers $\left(\eta_{1}, \eta_{2}, \eta_{3}\right)>0$ such that $\Omega\left(x_{0}, y_{0}, z_{0}\right) \subset R_{+}^{3}=\left\{(x, y, z): 0 \leq x \leq \eta_{1}, 0 \leq y \leq\right.$ $\left.\eta_{2}, 0 \leq z \leq \eta_{3}\right\}$ for all $\left(x_{0}, y_{0}, z_{0}\right)>0$, where $\Omega\left(x_{0}, y_{0}, z_{0}\right)$ is the omega limit set of the orbit initiating at $\left(x_{0}, y_{0}, z_{0}\right)$. Therefore, the system (3) is dissipative.

The following proposition provides a necessary condition for survival of the prey species $y$ in the system (3).

Proposition 1: A necessary condition for the prey species $y$ to survive is $1<\eta_{2}^{\beta}$.

Proof : From the second equation of the system (3) we get

$$
\begin{aligned}
\frac{d y}{d t}=b y & \left(1-y^{\beta}\right)-\frac{q y z}{1+b_{2} y+b_{3} z} \\
& \leq b y\left(1-y^{\beta}\right) \leq b y\left(1-\eta_{2}^{\beta}\right)=A y, \text { where } A=b \eta_{2}\left(1-\eta_{2}^{\beta}\right), \text { by the }
\end{aligned}
$$

theorem 1 .

Then we have $y \leq y_{0} e^{A t}$. Thus, for $A<0, \lim _{t \rightarrow \infty} y=0$. 
Thus the necessary condition for the servival of the prey species $y$ is $1<\eta_{2}^{\beta}$.

\subsection{Existence and stability criteria for equilibrium points}

The system (3) possesses the following equilibrium states:

1. The trivial state $E_{T} \equiv(0,0,0)$ always exists and it is with non-empty stable and unstable manifolds, so $E_{T}$ is unstable.

2. The axial state $E_{A} \equiv(1,0,0)$. It is also with non-empty stable and unstable manifolds, so $E_{A}$ is unstable.

3. The predator free equilibrium state is $\bar{E} \equiv(1,1,0)$.

The Jacobian matrix around $\bar{E}$ is given by

$$
J(\bar{E})=\left(\begin{array}{lll}
a_{11} & a_{12} & a_{13} \\
a_{21} & a_{22} & a_{23} \\
a_{31} & a_{32} & a_{33}
\end{array}\right)
$$

where,

$a_{11}=-a, a_{12}=0, a_{13}=-\frac{p}{1+b_{1}}, a_{21}=0, a_{22}=-b \beta, a_{23}=-\frac{q}{1+b_{2}}$, $a_{31}=\frac{\epsilon_{1} p}{\left(1+b_{1}\right)^{2}}, a_{32}=\frac{\epsilon_{1} p}{1+b_{1}}, a_{33}=-d-\frac{q}{1+b_{2}}$.

The characteristic equation of the Jacobian matrix $\bar{E}$ is given by

$$
\lambda^{3}+\Theta_{1} \lambda^{2}+\Theta_{2} \lambda+\Theta_{3}=0
$$

where,

$$
\begin{aligned}
\Theta_{1} & =-\left[a_{11}+a_{22}+a_{33}\right] \\
\Theta_{2} & =\left[\left(a_{22} a_{33}-a_{23} a_{32}\right)+\left(a_{11} a_{33}-a_{13} a_{31}\right)+a_{11} a_{22}\right] \\
\Theta_{3} & =-\left[a_{11}\left(a_{22} a_{33}-a_{23} a_{32}\right)-a_{13} a_{22} a_{31}\right]
\end{aligned}
$$

If $a_{33}>a_{13} a_{31}$, then $\Theta_{1}>0, \Theta_{3}>0$ and $\Theta_{1} \Theta_{2}-\Theta_{3}>0$ hold. Therefore we observe that the predator free equilibrium point $\bar{E}(1,1,0)$ of the system (3) is locally asymptotically stable if $a_{33}>a_{13} a_{31}$.

4. The interior equilibrium state $E^{*} \equiv\left(x^{*}, y^{*}, z^{*}\right)$, where $y^{*}\left(1-y^{* \beta}\right)=$ $\frac{a\left(1-x^{*}\right)\left[d+\left(d b_{1}-\epsilon_{1} p\right) x^{*}\right]}{p}, z^{*}=\frac{a\left(1+b_{1} x^{*}\right)\left(1-x^{*}\right)}{p}$ and $x^{*}$ is the positive root of the equation $A x^{* 3}+B x^{* 2}+C x^{*}+D=0$

where, $A=a \epsilon_{1} b_{1}, B=\epsilon_{1} a\left(1-b_{1}\right), C=d b_{1}\left(1-\epsilon_{2} b\right)+\epsilon_{1}\left(\epsilon_{2} b p-a\right), D=d\left(1-\epsilon_{2} b\right)$. The interior equilibrium point $E^{*}$ exists if $0<\frac{d}{\epsilon_{1} p-d b_{1}}<x^{*}<1$, when $p>\frac{d b_{1}}{\epsilon_{1}}$ is satisfied.

Now, we study the locally asymptotical stability criteria around the interior equilibrium state $E^{*}$. The Jacobian matrix around $E^{*}$ is given by

$$
J\left(E^{*}\right)=\left(\begin{array}{ccc}
A_{11} & A_{12} & A_{13} \\
A_{21} & A_{22} & A_{23} \\
A_{31} & A_{32} & A_{33}
\end{array}\right)
$$




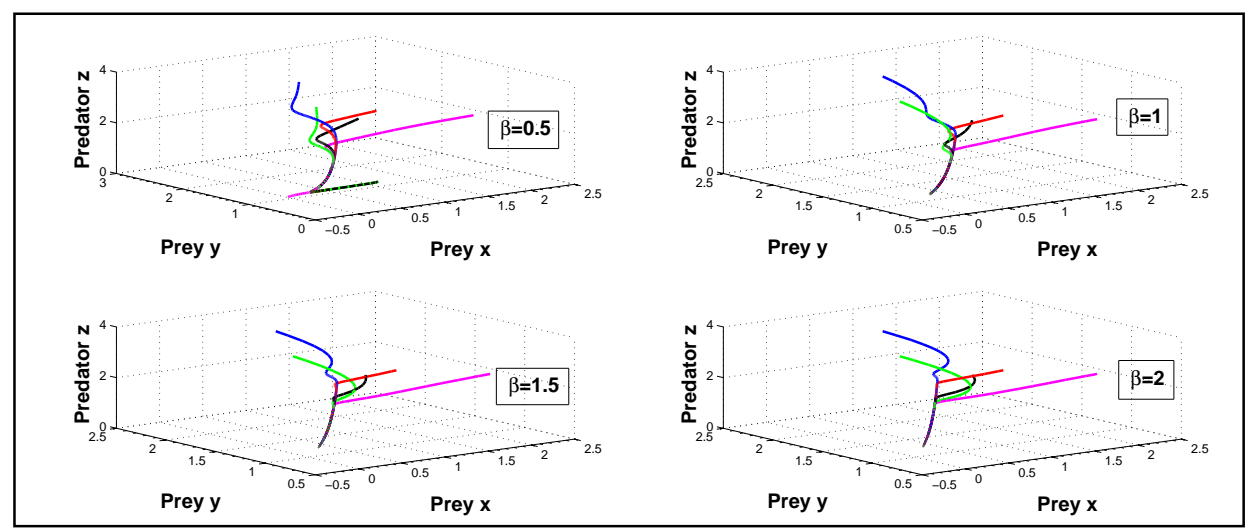

Figure 2: Phase portraits of the system (3) for $p=1.6, q=1.7, b_{1}=3$, $b_{2}=3.5, b_{3}=3.2, a=1, b=1, \epsilon_{1}=0.05, \epsilon_{2}=0.06, d=0.02$ and for different values of $\beta=0.5,1,1.5,2$.

where,

$A_{11}=a-2 a x^{*}-\frac{p z^{*}}{\left(1+b_{1} x^{*}\right)^{2}}, A_{12}=0, A_{13}=-\frac{p x^{*}}{1+b_{1} x^{*}}, A_{21}=0, A_{22}=$ $b\left[1-(\beta+1) y^{* \beta}\right]-\frac{q z^{*}\left(1+b_{3} z^{*}\right)}{\left(1+b_{2} y^{*}+b_{3} z^{*}\right)^{2}}, A_{23}=-\frac{q y^{*}\left(1+b_{2} y^{*}\right)}{\left(1+b_{2} y^{*}+b_{3} z^{*}\right)^{2}}, A_{31}=\frac{\epsilon_{1} p y^{*}}{\left(1+b_{1} x^{*}\right)^{2}}, A_{32}=$ $\frac{\epsilon_{1} p x^{*}}{1+b_{1} x^{*}}-\frac{q z^{*}\left(1+b_{3} z^{*}\right)}{\left(1+b_{2} y^{*}+b_{3} z^{*}\right)^{2}}, A_{33}=-d-\frac{q y^{*}\left(1+b_{2} y^{*}\right)}{\left(1+b_{2} y^{*}+b_{3} z^{*}\right)^{2}}$.

The characteristic equation of the Jacobian matrix $E^{*}$ is given by $\lambda^{3}+\Omega_{1} \lambda^{2}+\Omega_{2} \lambda+\Omega_{3}=0$

where,

$$
\begin{aligned}
& \Omega_{1}=-\left[A_{11}+A_{22}+A_{33}\right] \\
& \Omega_{2}=\left[\left(A_{22} A_{33}-A_{23} A_{32}\right)+\left(A_{11} A_{33}-A_{13} A_{31}\right)+A_{11} A_{22}\right] \\
& \Omega_{3}=-\left[A_{11}\left(A_{22} A_{33}-A_{23} A_{32}\right)-A_{13} A_{22} A_{31}\right]
\end{aligned}
$$

Using the Routh-Hurwitz criteria [18] we observe that the positive equilibrium point $E^{*}\left(x^{*}, y^{*}, z^{*}\right)$ of the system (3) is locally asymptotically stable if and only if $\Omega_{1}>0, \Omega_{3}>0$ and $\Omega_{1} \Omega_{2}-\Omega_{3}>0$ hold.

Now, we investigate the global stability of the interior equilibrium point $E^{*}$ of the system (3) in the following theorem.

Theorem 2: Suppose that the positive equilibrium point $E^{*}\left(x^{*}, y^{*}, z^{*}\right)$ is locally asymptotically stable. Then it is globally asymptotically stable if the following condition holds:

$$
M\left[\epsilon_{2} b+\frac{\epsilon_{1} p\left(x^{*}-z^{*}\right)}{1+b_{1} M}+\frac{\epsilon_{2} q\left(y^{*}-z^{*}\right)}{1+\left(b_{2}+b_{3}\right) M}+\epsilon_{2} b y^{*} M^{\beta-1}\right]<a \epsilon_{1} x^{*}+b \epsilon_{2} y^{*}-d z^{*}
$$




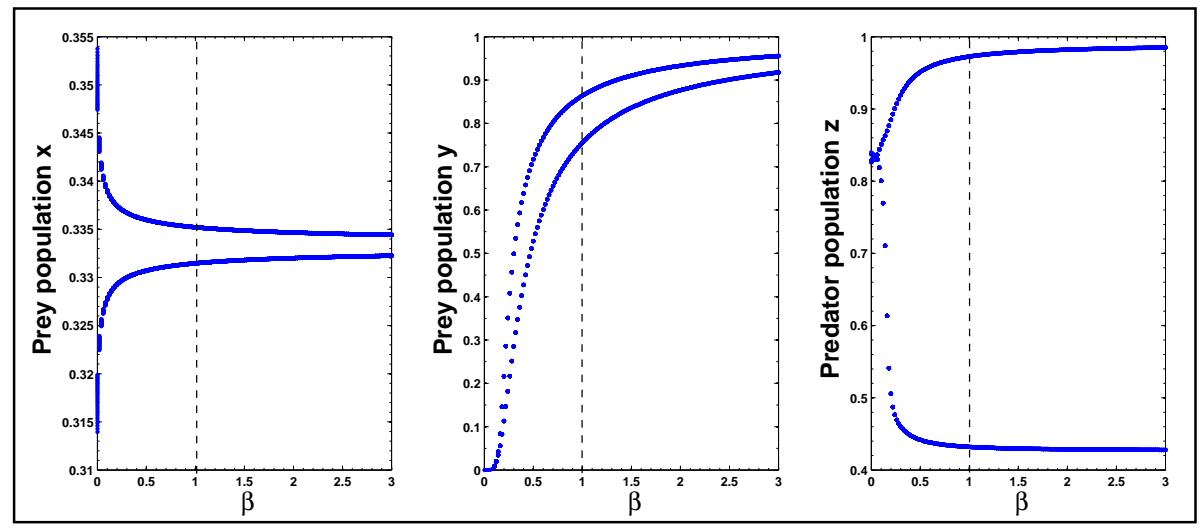

Figure 3: The bifurcation diagrams of the system (3) with respect to $\beta$ for $p=1.6, q=1.7, b_{1}=3, b_{2}=3.5, b_{3}=3.2, a=1, b=1, \epsilon_{1}=0.05, \epsilon_{2}=0.06$, $d=0.02$.

where $M=\frac{a \epsilon_{1}}{\theta}, \theta=\min \left\{a \epsilon_{1},-b \epsilon_{2}, d\right\}>0$.

Proof: The proof can be reached by using a Lyapunov stability theorem which gives a sufficient condition. Now, we consider a positive definite function about $E^{*}$ as

$$
W(x, y, z)=\epsilon_{1} W_{1}+\epsilon_{2} W_{2}+W_{3}
$$

where, $W_{1}(x, y, z)=x-x^{*} \ln \left(\frac{x}{x^{*}}\right), W_{2}(x, y, z)=y-y^{*} \ln \left(\frac{y}{y^{*}}\right), W_{3}(x, y, z)=$ $z-z^{*} \ln \left(\frac{z}{z^{*}}\right)$.

Therefore,

$$
\begin{gathered}
\frac{d W}{d t}=\epsilon_{1} \frac{\left(x-x^{*}\right)}{x} \frac{d x}{d t}+\epsilon_{2} \frac{\left(y-y^{*}\right)}{y} \frac{d y}{d t}+\frac{\left(z-z^{*}\right)}{z} \frac{d z}{d t} \\
\text { i.e., } \frac{d W}{d t}=\epsilon_{1}\left(x-x^{*}\right)\left\{a(1-x)-\frac{p z}{1+b_{1} x}\right\}+\epsilon_{2}\left(y-y^{*}\right)\left\{b\left(1-y^{\beta}\right)-\frac{q z}{1+b_{2} y+b_{3} z}\right\}+ \\
\left(z-z^{*}\right)\left\{\frac{\epsilon_{1} p x}{1+b_{1} x}+\frac{\epsilon_{2} q y}{1+b_{2} y+b_{3} z}-d\right\} .
\end{gathered}
$$

Using theorem (1), without loss of generality, we may assume that there exists a constant $M=\frac{a \epsilon_{1}}{\theta}$ satisfying $x(t), y(t), z(t)<M$, where $\theta=\min \left\{a \epsilon_{1},-b \epsilon_{2}, d\right\}>$ 0 and after algebraic calculation we have

$$
\begin{aligned}
& \quad \frac{d W}{d t} \leq-a \epsilon_{1}\left(x-\frac{1+x^{*}}{2}\right)^{2}-a \epsilon_{1} x^{*}-d z+\epsilon_{2} b M-\epsilon_{2} b y^{*}-\epsilon_{2} b y^{\beta+1}+\epsilon_{2} b y^{*} M^{\beta}+ \\
& \frac{\epsilon_{1} p M\left(x^{*}-z^{*}\right)}{1+b_{1} M}+\frac{\epsilon_{2} q M\left(y^{*}-z^{*}\right)}{1+\left(b_{2}+b_{3}\right) M}+d z^{*}-d z .
\end{aligned}
$$

It is easy to verify that $\frac{d W}{d t}<0$ under the condition (4). Therefore, $W$ is a lyapunov function with respect to $E^{*}$ in the interior positive octant. Hence, the equilibrium point $E^{*}\left(x^{*}, y^{*}, z^{*}\right)$ is globally asymptotically stable. 


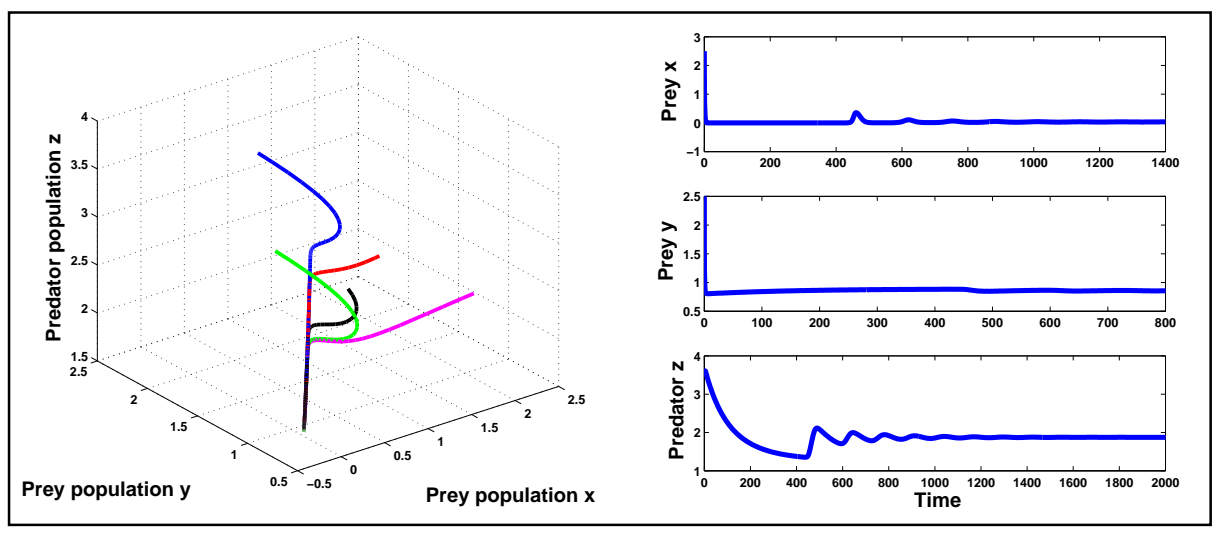

Figure 4: Global dynamics of the system (5) for $p=1.6, q=1.7, b_{1}=3$, $b_{2}=3.5, b_{3}=3.2, a=1, b=1, c_{1}=0.1, c_{2}=0.12, \epsilon_{1}=0.05, \epsilon_{2}=0.06$, $d=0.02, \alpha=2, \xi=1$ and $\beta=2$.

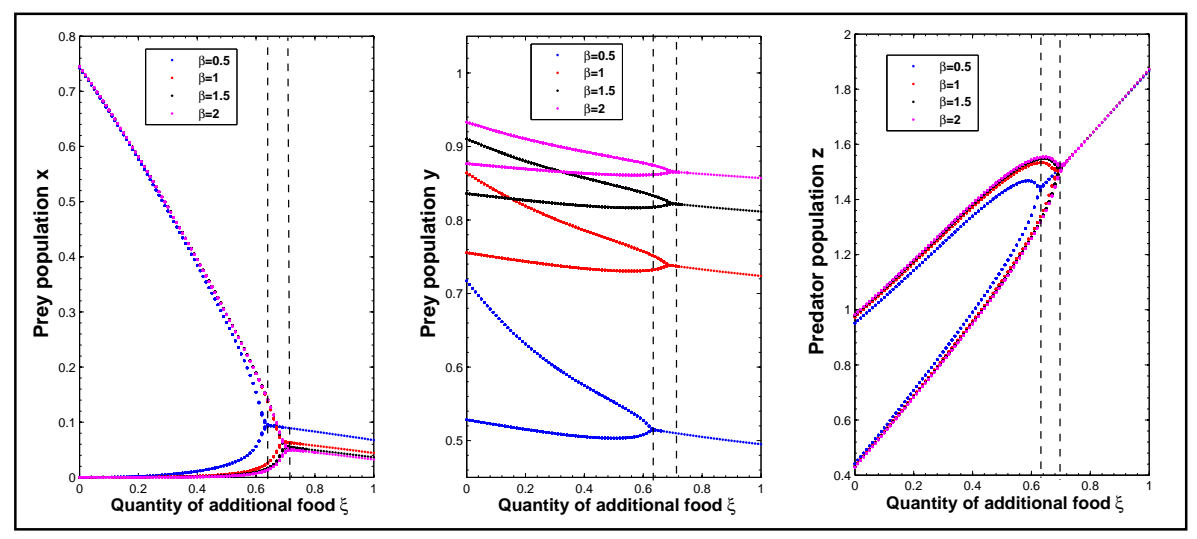

Figure 5: The bifurcation diagrams of the system (5) with respect to quantity of additional food $\xi$ for $\alpha=2, p=1.6, q=1.7, b_{1}=3, b_{2}=3.5, b_{3}=3.2$, $a=1, b=1, c_{1}=0.1, c_{2}=0.12, \epsilon_{1}=0.05, \epsilon_{2}=0.06, d=0.02$ and for different values of $\beta=0.5,1,1.5,2$. 


\section{Dynamics with additional food}

Now we are interested to study the dynamics of the predator-prey system (3) with additional food to predator. From this study, it is possible to develope management strategies that manipulate quality of the additional food (characterized by predator's handling time ) and its supply level for the benefit of biological control [19-21]. We will also able to understand the indirect interactions taking place in the ecosystem and their vital role on the predator, which helps us to predict the eventual state of the ecosystem and predator[2224]. Here we assume that the number of encounters per predator with the additional food is proportional to the density of the additional food and the proportionality constant characterizes the ability of the predator to identify the additional food. So, we introduce the additional food (characterized by predator's handling time ) using the constant term $\alpha \xi[19]$ to the predator. The term $\alpha$ denotes the quality of additional food and $\xi$ denotes quantity of additional food [19]. Therefore, using this term we reformulate the system (3) in the following form

$$
\begin{aligned}
\frac{d x}{d t} & =a x(1-x)-\frac{p x z}{1+\alpha \xi+b_{1} x} \\
\frac{d y}{d t} & =b y\left(1-y^{\beta}\right)-\frac{q y z}{1+\alpha \xi+b_{2} y+b_{3} z} \\
\frac{d z}{d t} & =\frac{\epsilon_{1} p\left(x+c_{1} \xi\right) z}{1+\alpha \xi+b_{1} x}+\frac{\epsilon_{2} q\left(y+c_{2} \xi\right) z}{1+\alpha \xi+b_{2} y+b_{3} z}-d z
\end{aligned}
$$

The main objective of this section is to study the dynamics of the system (5) in presence of additional food. We are interested to investigate the influence of additional food on a system. In this paper, we have performed a numerical simulation to investigate the influence of additional food in a system (5).

\section{$4 \quad$ Numerical results}

We have done numerical simulation of the system (3) with parameters values $p=1.6, q=1.7, b_{1}=3, b_{2}=3.5, b_{3}=3.2, a=1, b=1, \epsilon_{1}=0.05$, $\epsilon_{2}=0.06, d=0.02$ which are fixed through out the simulation.

Figure 1 is the phase portraits of the system (3) for different values of $\beta$. From figure 1, we observe that the system (3) is stable about its equilibrium point for higher values of $\beta$. We have done bifurcation analysis of the system (3) with respect to $\beta$ is shown in figure 2 . From figure 2 , we observe that the prey population $x$ has stable limit cycle, while the prey populatin $y$ extinct for very low values of $0<\beta<0.1$ and for higher values of $\beta$ it also has stable limit 
cycle dynamics. Predator population $z$ has stable dynamics depending on the values of $\beta$. From figure 2 we observe that the density of predator population is high after $\beta>1$. Therefore for higher values of $\beta$, the systems dynamics shows stable limit cycle behaviour. From figure 2 it is observe that population density depends on the values of $\beta$.

For the system (5), we have done phase portrait diagrams and bifurcation analysis for different supply of additional food. Figure 3 shows the stablity of the system (5) in presence of additional food with $\alpha=2$ and $\xi=1$. Bifurcation analysis of the system (5) with respect to quantity of additional food $\xi$ is done for different values of $\beta$ which is shown in the figure 4 . Figure 4 shows that the system (5) has limit cycle oscillations in some specific range of $\xi$ and then the system (5) settles down to steady state. Therefore, the system is effected with the supply of additional food as well as the different value of $\beta$. From the numerical simulation we conclude that the stable coexistance of a food chain system depends on the growth rate of the species.

\section{Conclusion}

We have proposed a predator-prey model with different growth rates and different functional responces of the species. We have done global stability under certain condition of the proposed model. We have also shown that the system has limit cycle oscillation and stable coexisting dynamics for different growth rates. We also have shown the influence of additional food on the system for different growth rates and different consumption rates. From our analysis, we have shown that the prey species has extinction risk for lower values of $\beta$. Therefore, servival of species depends on growth rates and consumption rates. But supply of additional food to predator be helpful for survival of prey species. Therefore, a real predator-prey model should be constructed with different growth rates and different functional responces for the existence of prey species. Therefore, a food chain system with different types of functional re-

sponses and different growth rates for preys may be able to achieve the typical behaviour of real food chain.

\section{ACKNOWLEDGEMENTS}

I am grateful to the Editor for careful reading and constructive comments. 


\section{References}

[1] P.F. Verhulst, Notice sur la loi que la population suit dans son accroissement, Corr. Math. Physics, 10 (1838) 113.

[2] P.C.J. van Rijn, Y.M. van Houten, M.W. Sabelis, How plants benefit from providing food to predators even when it is also edible to herbivores, Ecology 83 (2002) 2664-2679.

[3] R.Pearl, The growth of populations, Quarterly Review of Biology 2 (1927) 532-548.

[4] B.J.T. Morgan, Stochastic models of groupings changes, Advances in Applied Probability 8 (1976) 30-57.

[5] T.C.Fisher, R.H.Fry, Tech. Forecasting Soc. Changes 3 (1971) 75.

[6] R.Herman, E.W.Montroll, Proceedings of the National Academy of Sciences, USA 69 (1972) 3019.

[7] R.Pearl, L.J.Reed, On the rate of growth of the population of United States since 1790 and its mathematical representation, Proceedings National Academy of Sciences USA, G (1920) 275.

[8] F.J.Richards, A Flexible Growth Function for Empirical Use, Journal of Experimental Botany 10(29) (1959) 290-300.

[9] L.Von.Bertalanffy, A quantitative theory of organic growth, Human Biology 10(2) (1938) 181-213.

[10] J.A.Nelder, The fitting of a generalization of the logistic curve, Biometrics 17 (1961) 89-110.

[11] S. Gakkhar, A. singh Control of Chaos due to additional predator in the Hasting-Powell food chain model, J Math. Anal. Appl. 385 (2012) 423-438.

[12] T.K. Kar, H. Matsuda, Global dynamics and controllability of a harvested prey-predator system with Holling type III functional response, Nonlinear Anal: Hybrid system 1 (2007) 59-67.

[13] Y. Do, H. Baek, Y. Lim , D. Lim, A three species food chain system with two types of functional responses, Abstract and Applied Analysis, vol 2011, doi:10.1155/2011/934569.

[14] M. Fan, Y. Kuang, Dynamics of a nonautonomous predator-prey system with Beddington-DeAngelis functional response, Journal of Mathematical Analysis and applications 295 (2004) 15-39. 
[15] B. Patra, A. Maiti, G. P. Samanta, Effect of Time-Delay on a RatioDependent Food Chain Model, Nonlinear Analysis: Modelling and Control 14 (2009) 199-216.

[16] Dongmei Xiao, Wenxia Li, Maoan Han, Dynamics in a ratio-dependent predatorprey model with predator harvesting, J. Math. Anal. Appl. 324 (2006) 14-29.

[17] H.I. Freedman, Q. Hongshun, Interactions leading to persistence in predator-prey system with group defense, Bull. Math. Biol. 50 (1988) 517-530.

[18] H. Freedman, P. Waltman, Persistent in models of three interacting predator-prey populations, Math. Biosci. 68 (1984) 213-231.

[19] P.D.N. Srinivasu, B.S.R.V. Prasad, M. Venkatesulu, Biological control through provision of additional food to predators:a theoretical study, Theor Popul Biol. 72 (2007) 111-120.

[20] B. Sahoo, S. Poria, Dynamics of a Predator-prey System with Seasonal Effects on Additional Food, Int. J. Ecosy. 1 (2011) 10-13.

[21] B. Sahoo, A Predator-Prey Model with General Holling Interactions in Presence of Additional Food, Int. J. Plant Research. 2 (2012) 47-50.

[22] M. Coll, M. Guershon, Omnivoryin terrestrial arthropods: mixing plant and prey diets, Annu. Rev. Entomol. 47 (2002) 267-297.

[23] Holt, R.D., Lawton, J.H., The ecological consequences of shared natural enemies, Annu. Rev. Ecol. Syst. 25 (1994) 495-520.

[24] Wootton, J.T., The nature and consequences of indirect effects in ecological communities, Annu. Rev. Ecol. Syst. 25 (1994) 443-466. 